\title{
Correction to: Legal Aspects of Offshore CCS: Case Study - Salt Cavern
}

\section{Hirdan K. M. Costa ${ }^{1}$ Raíssa M. L. M. Musarra ${ }^{1} \cdot$ Isabela Morbach Machado e Silva ${ }^{1} \cdot$ Romário de Carvalho Nunes $^{1}$.} Israel Lacerda Cavalcante ${ }^{1} \cdot$ Silvia Andrea Cupertino ${ }^{1} \cdot$ Robert Darnowsky $^{1}$

Published online: 3 January 2020

(C) Escola Politécnica - Universidade de São Paulo 2020

Correction to: Polytechnica (2019) 2:87-96.

https://doi.org/10.1007/s41050-019-00011-4

The author named Israel Lacerda Cavalcante should be cited as Israel Lacerda de Araújo or ARAÚJO, Israel L. in all the publications of this article.

The online version of the original article can be found at https://doi.org/ 10.1007/s41050-019-00011-4

Robert Darnowsky

robert.darnowsky@springer.com

Hirdan K. M. Costa

hirdan@usp.br

Raíssa M. L. M. Musarra

raissa@musarra.com.br

Isabela Morbach Machado e Silva

isabelamorbach@gmail.com

Romário de Carvalho Nunes marioviolino@yahoo.com.br

Israel Lacerda Cavalcante israel@senado.leg.br

Silvia Andrea Cupertino cupertino@usp.br

1 Energy and Environment Institute, São Paulo University (Instituto de Energia e Ambiente IEE/USP), São Paulo, Brazil 